\title{
KOROUS TYPE INEQUALITIES FOR ORTHOGONAL POLYNOMIALS IN TWO VARIABLES
}

\author{
BRANISLAV FTOREK — PAVOL ORŠANSKÝ
}

\begin{abstract}
J. Korous reached an important result for general orthogonal polynomials in one variable. He dealt with the boundedness and uniform boundedness of polynomials $\left\{P_{n}(x)\right\}_{n=0}^{\infty}$ orthonormal with the weight function

$$
h(x)=\delta(x) \widetilde{h}(x),
$$

where $\widetilde{h}(x)$ is the weight function of another system of polynomials $\left\{\widetilde{P}_{n}(x)\right\}_{n=0}^{\infty}$ orthonormal in the same interval and

$$
\delta(x) \geq \delta_{0}>0
$$

is a certain function. We generalize this result for orthogonal polynomials in two variables multiplying their weight function $h(x, y)$ by a polynomial, dividing $h(x, y)$ by a polynomial, and multiplying $h(x, y)$ with separated variables by a certain function $\delta(x, y)$.
\end{abstract}

\section{Introduction}

A generalization of the weight function and a study of relevant properties of orthogonal polynomials are important parts of investigation in the theory of orthogonal polynomials. J. K or o u s reached an important result for general orthogonal polynomials in one variable in [6]. The theorem first published in that paper later became known as Korous theorem.

THEOREM 1.1 (Korous). Let a system of polynomials $\left\{\widetilde{P}_{n}(x)\right\}_{n=0}^{\infty}$ orthonormal with a weight function $\widetilde{h}(x)$ and generalized system of polynomials $\left\{P_{n}(x)\right\}_{n=0}^{\infty}$

(C) 2014 Mathematical Institute, Slovak Academy of Sciences.

2010 Mathematics Subject Classification: 33C45, 33C50, 33D50.

Keywords: Korous theorem, orthogonal polynomials in two variables, weight function, boundedness.

The authors gratefully acknowledge the Scientific Grant Agency VEGA of the Ministry of Education of Slovak Republic and the Slovak Academy of Sciences for supporting this work under the Grant No. 1/1245/12. 


\section{BRANISLAV FTOREK — PAVOL ORŠANSKÝ}

orthonormal with a weight function $h(x)=\widetilde{h}(x) \delta(x)$ be given, where

$$
\delta(x) \geq \delta_{0}>0 \quad \text { and } \quad \delta(x)
$$

satisfies the Lipschitz condition

$$
\left|\delta\left(x_{1}\right)-\delta\left(x_{2}\right)\right| \leq M\left|x_{1}-x_{2}\right|, \quad x_{1}, x_{2} \in(a, b),
$$

$(a, b)$ is the interval of orthogonality of the polynomials

$$
\left\{\widetilde{P}_{n}(x)\right\}_{n=0}^{\infty} \text { and }\left\{P_{n}(x)\right\}_{n=0}^{\infty} .
$$

Then the following estimation

$$
\left|P_{n}(x)\right| \leq \frac{1}{\delta_{0}}\left|\widetilde{P}_{n}(x)\right|+\frac{K M}{\delta_{0}^{3 / 2}}\left(\left|\widetilde{P}_{n}(x)\right|+\left|\widetilde{P}_{n-1}(x)\right|\right),
$$

where $\delta_{0}=\min \delta(x), x \in(a, b)$ and $K=\max \{|a|,|b|\}$ holds.

In the paper, we address this topic to orthogonal polynomials in two variables. However, the application of certain techniques from the field of orthogonal polynomials in one variable is not possible. For example, a reproducing kernel function for orthogonal polynomials in two variables $K_{n}(x, y, u, v)$ has a complex integral form (see (4.10) and the monograph [11]), as opposed to the simple relation (4.3). The expression (4.3) of the kernel $K_{n}(x, t)$ plays a fundamental role in the original proof of the Korous theorem. Therefore, we used some simplifying assumptions and the obtained results are not quite general.

\subsection{Orthogonal polynomials in two variables}

Many authors dealt with the issues associated with the orthogonal polynomials in two variables, e.g., J a c k s o n ([3]), K o or n w i n d e r (44, [5]), M a r čo ková ([8], [9], [10]), Krall, Sheffer ([7]) and Suetin ([1]). We recall some properties of the algebraic polynomials in two wariables given in [1]. Let an algebraic polynomial in two variables be written in the form

$$
P_{n, k}(x, y)=\sum_{m=0}^{n-1} \sum_{s=0}^{m} c_{m, s} x^{m-s} y^{s}+\sum_{s=0}^{k} c_{n, s} x^{n-s} y^{s} .
$$

Index $n$ in this formula is a total degree of the polynomial with respect to the variables $x$ and $y$. The second sum in relation (1.1) contains the homogeneous monomials of the total degree $n$ with respect to the variables $x$ and $y$. Thus, we get the leading coefficients

$$
c_{n, 0}, c_{n, 1}, \ldots, c_{n, k-1}, c_{n, k}
$$

The last of these coefficients, i.e., the number $c_{n, k}$, is called the principal coefficient of the polynomial (1.1). It is natural to assume that the principal coefficient is not equal to zero. In this case we say that the polynomial (1.1) has an order $(n, k)$. Obviously, some of the main coefficients in (1.2), except for the principal one, may be equal to zero. 
The first sum in the relation (1.1) contains at most $\frac{1}{2} n(n+1)$ terms, and the second one at most $(k+1)$ terms. This means that the number of all terms of the polynomial is

$$
N(n, k)=\frac{n(n+1)}{2}+k+1 .
$$

We say that the order $(r, s)$ of the polynomial $P_{r, s}(x, y)$ is lower than the order $(n, k)$ of (1.1), if $r<n$ or $r=n$ and $s<k$. We denote it by $(r, s)<(n, k)$.

Lemma 1.2 ([11, Lemma 1]). Let every polynomial of the system $\left\{P_{n, k}(x, y)\right\}$ have a nonzero principal coefficient. Then, any polynomial $Q_{n, k}(x, y)$ of the order $(n, k)$ can be uniquely represented in the form

$$
Q_{n, k}(x, y)=\sum_{m=0}^{n-1} \sum_{s=0}^{m} a_{m, s} P_{m, s}(x, y)+\sum_{s=0}^{k} a_{n, s} P_{n, s}(x, y) .
$$

Definition 1.3 (11]). Let $G$ be a finite simply connected domain in the plane $x O y$ which is bounded by a rectifiable Jordan curve $\Gamma$. A non-negative function $h(x, y)$ is called a weight function in the domain $G$, if it is integrable over the domain $G$ and is not equivalent to zero, i.e., the condition

holds.

$$
0<\iint_{G} h(x, y) d x d y<\infty
$$

Definition 1.4 (11]). The system of algebraic polynomials $\left\{F_{n, k}(x, y)\right\}$ is called orthonormal over the domain $G$ with the weight function $h(x, y)$, if the following conditions are fulfilled:

(i) the principal coefficient $c_{n, k}$ of every polynomial $F_{n, k}(x, y)$ is positive,

(ii) polynomials of the system $\left\{F_{n, k}(x, y)\right\}$ satisfy the condition of orthonormality with the weight $h(x, y)$ over the domain $G$, i.e.,

$$
\iint_{G} h(x, y) F_{n, k}(x, y) F_{m, s}(x, y) d x d y=\delta_{n, m} \delta_{k, s},
$$

where $\delta_{i, j}$ is the Kronecker delta.

In the Definition 1.3. the conditions of boundedness of the domain $G$ and of rectifiability of the contour $\Gamma$ may be omitted. Then, we require the existence of all power moments of the weight function $h(x, y)$, i.e., integrals

must be finite.

$$
h_{n, k}=\iint_{G} h(x, y) x^{n-k} y^{k} d x d y
$$




\section{BRANISLAV FTOREK — PAVOL ORŠANSKÝ}

LEMma 1.5 ([11, Theorem 1]). Let a polynomial $F_{n, k}(x, y)$ of order $(n, k)$ with the principal coefficient $c_{n, k} \neq 0$ be given. This polynomial is an orthogonal polynomial with a weight function $h(x, y)$ over a domain $G$ if and only if for any polynomial $P_{p, q}(x, y)$ of lower order $(p, q)$ the following condition

holds.

$$
\iint_{G} h(x, y) F_{n, k}(x, y) P_{p, q}(x, y) d x d y=0
$$

\section{Multiplying weight function with a polynomial}

Let $R_{r, t}(x, y) \geq 0$ be a polynomial defined over the domain $G$, where $G$ is an interior of a closed rectifiable Jordan curve $\Gamma$. Let $P_{n, k}(x, y)$ be a polynomial orthonormal on the domain $G$ with respect to the weight function $h(x, y)$. Further, let $Q_{n, k}(x, y)$ be a polynomial orthonormal on the domain $G$ with respect to the weight function

$$
w(x, y)=R_{r, t}(x, y) h(x, y) .
$$

The total degree of the product $R_{r, t}(x, y) Q_{n, k}(x, y)$ will not be greater than $(n+r)$, and according to (1.4), we have

where

$$
R_{r, t}(x, y) Q_{n, k}(x, y)=\sum_{m=0}^{n+r} \sum_{s=0}^{m} c_{m, s} P_{m, s}(x, y),
$$

$$
c_{m, s}=\iint_{G} h(x, y) R_{r, t}(x, y) Q_{n, k}(x, y) P_{m, s}(x, y) d x d y .
$$

If $m<n$, from the condition of orthogonality of $Q_{n, k}(x, y)$ on domain $G$ with respect to the weight function (2.1), we get $c_{m, s}=0$ (Lemma 1.5), and the polynomial (2.2) has a form

$$
R_{r, t}(x, y) Q_{n, k}(x, y)=\sum_{m=n}^{n+r} \sum_{s=0}^{m} c_{m, s} P_{m, s}(x, y) .
$$

Using Schwarz inequality for non-zero coefficients $c_{m, s}$, we have

$$
c_{m, s}^{2} \leq \iint_{G} h(x, y) R_{r, t}^{2}(x, y) Q_{n, k}^{2}(x, y) d x d y \iint_{G} h(x, y) P_{m, s}^{2}(x, y) d x d y .
$$

Since $P_{m, s}(x, y)$ is the polynomial orthonormal on the domain $G$ with respect to the weight function $h(x, y)$, the second integral in the above relationship is equal to one. Let $K$ be a maximum of the polynomial $R_{r, t}(x, y)$ on the domain $G$. 


\section{KOROUS TYPE INEQUALITIES}

Then,

$$
\begin{aligned}
& \iint_{G} h(x, y) R_{r, t}^{2}(x, y) Q_{n, k}^{2}(x, y) d x d y \leq \\
& \leq K \iint_{G} h(x, y) R_{r, t}(x, y) Q_{n, k}^{2}(x, y) d x d y=K,
\end{aligned}
$$

because $Q_{n, k}(x, y)$ is an orthonormal polynomial on the domain $G$ with respect to the weight function (2.1). So,

$$
\left|c_{m, s}\right| \leq K^{1 / 2}
$$

Based on the above considerations, we state the following theorem.

THEOREM 2.1. Let the polynomial $R_{r, t}(x, y) \geq 0$ on the finite simply connected domain $G$. Let $\left\{P_{n, k}(x, y)\right\}$ be a system of polynomials orthonormal on the domain $G$ with respect to the weight function $h(x, y)$. Let $Q_{n, k}(x, y)$ be a polynomial orthonormal on the domain $G$ with respect to the weight function

$$
w(x, y)=R_{r, t}(x, y) h(x, y)
$$

and let

$$
\left|P_{n, k}(x, y)\right| \leq H
$$

on the domain $G$, where $H$ does not depend on the total degree of these polynomials and on variables $x, y$.

Then,

$$
\left|R_{r, t}(x, y) Q_{n, k}(x, y)\right| \leq \frac{1}{2}(r+1)(2 n+r+2) K^{1 / 2} H
$$

on the domain $G$, where $K$ is the maximum of the polynomial $R_{r, t}(x, y)$ on $G$.

Proof. From (1.3), we have $N(n, n)=\frac{(n+1)(n+2)}{2}$, and the expression (2.4) has at most

$$
\begin{aligned}
N(n+r, n+r)-N(n-1, n-1) & =\frac{(n+r+1)(n+r+2)}{2}-\frac{n(n+1)}{2} \\
& =\frac{1}{2}(r+1)(2 n+r+2)
\end{aligned}
$$

terms. And now, the inequality (2.7) follows from (2.4), (2.5) and (2.6).

\section{Dividing weight function with a polynomial}

Let $R_{r, t}(x, y)>0$ be a polynomial defined over the domain $G$, where $G$ is an interior of the closed rectifiable Jordan curve $\Gamma$. Let $P_{n, k}(x, y)$ be a polynomial orthonormal on the domain $G$ with respect to the weight function $h(x, y)$. 


\section{BRANISLAV FTOREK — PAVOL ORŠANSKÝ}

Next, let $Q_{n, k}(x, y)$ be a polynomial orthonormal on the domain $G$ with respect to the weight function

$$
w(x, y)=\frac{h(x, y)}{R_{r, t}(x, y)} .
$$

In the sum

$$
Q_{n, k}(x, y)=\sum_{m=0}^{n} \sum_{s=0}^{m} c_{m, s} P_{m, s}(x, y),
$$

coefficients $c_{m, s}$ may be written in the form

$$
c_{m, s}=\iint_{G} \frac{h(x, y)}{R_{r, t}(x, y)} Q_{n, k}(x, y) P_{m, s}(x, y) R_{r, t}(x, y) d x d y .
$$

For $m+r<n$, these integrals are equal to zero (according to Lemma 1.5), because the polynomial $P_{m, s}(x, y) R_{r, t}(x, y)$ has the total degree less than $n$. Then, we have

$$
Q_{n, k}(x, y)=\sum_{m=n-r}^{n} \sum_{s=0}^{m} c_{m, s} P_{m, s}(x, y) .
$$

For $r>n$, we get (according to (3.2))

$$
Q_{n, k}(x, y)=\sum_{m=0}^{n} \sum_{s=0}^{m} c_{m, s} P_{m, s}(x, y) .
$$

For the nonzero coefficients in these sums, we get

$$
\left|c_{m, s}\right| \leq \iint_{G} h(x, y)\left|Q_{n, k}(x, y)\right|\left|P_{m, s}(x, y)\right| d x d y .
$$

We denote by $K$ the maximum of the polynomial $R_{r, t}(x, y)$ on $G$. Using the Schwarz inequality again and using the orthonormal property of the polynomials $P_{m, s}(x, y), Q_{n, k}(x, y)$, we get

$$
\begin{aligned}
c_{m, s}^{2} & \leq \iint_{G} h(x, y) Q_{n, k}^{2}(x, y) d x d y \iint_{G} h(x, y) P_{m, s}^{2}(x, y) d x d y \\
& =\iint_{G} h(x, y) Q_{n, k}^{2}(x, y) d x d y \\
& =\iint_{G} \frac{h(x, y)}{R_{r, t}(x, y)} R_{r, t}(x, y) Q_{n, k}^{2}(x, y) d x d y \\
& \leq K \iint_{G} \frac{h(x, y)}{R_{r, t}(x, y)} Q_{n, k}^{2}(x, y) d x d y=K .
\end{aligned}
$$

Therefore,

$$
\left|c_{m, s}\right| \leq K^{1 / 2}
$$


TheOREM 3.1. Let the polynomial $R_{r, t}(x, y)>0$ on the finite simply connected domain $G$. Let $\left\{P_{n, k}(x, y)\right\}$ be a system of polynomials orthonormal on the domain $G$ with respect to the weight function $h(x, y)$. Let $Q_{n, k}(x, y)$ be a polynomial orthonormal on the domain $G$ with respect to the weight function

$$
w(x, y)=\frac{h(x, y)}{R_{r, t}(x, y)},
$$

and let

$$
\left|P_{n, k}(x, y)\right| \leq H
$$

on the domain $G$, where $H$ does not depend on the total degree of this polynomial and on the variables $x, y$. Then, the following inequalities hold:

$$
\begin{array}{ll}
\text { if } r<n, & \left|Q_{n, k}(x, y)\right| \leq \frac{1}{2}(r+1)(2 n-r+2) K^{1 / 2} H, \\
\text { if } r \geq n, & \left|Q_{n, k}(x, y)\right| \leq \frac{1}{2}(n+1)(n+2) K^{1 / 2} H .
\end{array}
$$

$K$ is the maximum of the polynomial $R_{r, t}(x, y)$ on $G$ and $(x, y) \in G$.

P r o o f. The sum (3.4) has at most (according to (1.3))

$$
\begin{aligned}
N(n, n)-N(n-r-1, n-r-1) & =\frac{(n+1)(n+2)}{2}-\frac{(n-r)(n-r+1)}{2} \\
& =\frac{1}{2}(r+1)(2 n-r+2)
\end{aligned}
$$

terms and the sum (3.5) has at most $N(n, n)=\frac{1}{2}(n+1)(n+2)$ terms. It is obvious that (3.9), (3.10) follow from (3.4), (3.5), (3.6) and (3.8).

Observe that a similar problem and related topics have been investigated in [1] for orthogonal polynomials in one variable. The connection coefficients between two sets of orthogonal polynomials on the real line are studied in the paper [12].

\section{Weight function with separated variables}

In this section, we assume that the weight function has separated variables (cf. 4], [5], [9], [10]), i.e.,

$$
h(x, y)=v_{1}(x) v_{2}(y) .
$$

Let the polynomials $\left\{p_{n}(x)\right\}$ be orthonormal on an interval $(a, b)$ with a weight function $v_{1}(x)$, i.e.,

$$
\int_{a}^{b} v_{1}(x) p_{n}(x) p_{m}(x) d x=\delta_{n, m}, \quad-\infty \leq a<b \leq \infty,
$$




\section{BRANISLAV FTOREK — PAVOL ORŠANSKÝ}

and let the system of orthonormal polynomials $\left\{q_{n}(x)\right\}$ be associated with a weight function $v_{2}(x)$ on $(c, d)$, i.e.,

$$
\int_{c}^{d} v_{2}(x) q_{n}(x) q_{m}(x) d x=\delta_{n, m}, \quad-\infty \leq c<d \leq \infty .
$$

Consider now the polynomials in two variables

$$
F_{n, m}(x, y)=p_{n-m}(x) q_{m}(y) .
$$

These polynomials are orthonormal over the domain $G=(a, b) \times(c, d)$ with respect to the weight function (4.1).

For polynomials $p_{n}(x)=a_{n}^{(n)} x^{n}+\cdots$ and $q_{n}(x)=b_{n}^{(n)} x^{n}+\cdots$, we have reproducing kernel functions given by the Christoffel-Darboux formulae (cf. [13, Theorem 3.2.2])

$$
K_{n}(x, t)=\sum_{i=0}^{n} p_{i}(x) p_{i}(t)=\frac{a_{n}^{(n)}}{a_{n+1}^{(n+1)}} \frac{p_{n+1}(x) p_{n}(t)-p_{n}(x) p_{n+1}(t)}{x-t}
$$

and

$$
K_{n}^{*}(x, t)=\sum_{i=0}^{n} q_{i}(x) q_{i}(t)=\frac{b_{n}^{(n)}}{b_{n+1}^{(n+1)}} \frac{q_{n+1}(x) q_{n}(t)-q_{n}(x) q_{n+1}(t)}{x-t} .
$$

Letting $t \rightarrow x$, we find

$$
K_{n}(x, x)=\sum_{i=0}^{n} p_{i}(x) p_{i}(x)=\frac{a_{n}^{(n)}}{a_{n+1}^{(n+1)}}\left[p_{n+1}^{\prime}(x) p_{n}(x)-p_{n}^{\prime}(x) p_{n+1}(x)\right]
$$

and

$$
K_{n}^{*}(x, x)=\sum_{i=0}^{n} q_{i}(x) q_{i}(x)=\frac{b_{n}^{(n)}}{b_{n+1}^{(n+1)}}\left[q_{n+1}^{\prime}(x) q_{n}(x)-q_{n}^{\prime}(x) q_{n+1}(x)\right] .
$$

THEOREM 4.1. Let $\left\{F_{n, m}(x, y)\right\}$ be a system of polynomials (4.2) orthonormal with the weight function $h(x, y)=v_{1}(x) v_{2}(y)$ over the domain $G=(a, b) \times(c, d)$. Let $\left\{Q_{n, m}(x, y)\right\}$ be a system of orthonormal polynomials associated with the weight function

$$
w(x, y)=h(x, y) \delta(x, y)
$$

on $G$. Let the function $\delta(x, y)$ be bounded on $G$ and

$$
\delta(x, y) \geq M>0 \text {. }
$$

Then, the following estimation

$$
\left|Q_{n, k}(x, y)\right| \leq \frac{1}{\sqrt{M}} \sum_{s=0}^{k}\left|F_{n, s}(x, y)\right|+\frac{n}{\sqrt{M}}\left(K_{n-1}(x, x) K_{n-1}^{*}(y, y)\right)^{\frac{1}{2}}
$$

holds on $G$. The kernels $K_{n}(x, x), K_{n}^{*}(x, x)$ are given by (4.4) and (4.5). 


\section{KOROUS TYPE INEQUALITIES}

P r o o f. The polynomial $Q_{n, k}(x, y)$ can be represented in the form (Lemma 1.2)

$$
Q_{n, k}(x, y)=\sum_{m=0}^{n-1} \sum_{s=0}^{m} c_{m, s} F_{m, s}(x, y)+\sum_{s=0}^{k} c_{n, s} F_{n, s}(x, y)
$$

where the coefficients $c_{i, j}$ are determined by the equality

$$
c_{i, j}=\iint_{G} h(u, v) Q_{n, k}(u, v) F_{i, j}(u, v) d u d v .
$$

Substituting (4.9) into the expansion (4.8), we obtain

$$
\begin{aligned}
Q_{n, k}(x, y)= & \iint_{G} h(u, v) Q_{n, k}(u, v)\left[\sum_{m=0}^{n-1} \sum_{s=0}^{m} F_{m, s}(x, y) F_{m, s}(u, v)\right] d u d v \\
& +\iint_{G} h(u, v) Q_{n, k}(u, v)\left[\sum_{s=0}^{k} F_{n, s}(x, y) F_{n, s}(u, v)\right] d u d v .
\end{aligned}
$$

The sum

$$
K_{n}(x, y, u, v)=\sum_{m=0}^{n} \sum_{s=0}^{m} F_{m, s}(x, y) F_{m, s}(u, v),
$$

similarly to the case of orthonormal polynomials in one variable, there is called the kernel of order $n$ of the orthonormal polynomials system (cf. [11]). Then, we have

$$
\begin{aligned}
Q_{n, k}(x, y)= & \iint_{G} h(u, v) Q_{n, k}(u, v) K_{n-1}(x, y, u, v) d u d v \\
& +\iint_{G} h(u, v) Q_{n, k}(u, v)\left[\sum_{s=0}^{k} F_{n, s}(x, y) F_{n, s}(u, v)\right] d u d v .
\end{aligned}
$$

If we denote the first integral in previous relation

$$
S_{1}=\iint_{G} h(u, v) Q_{n, k}(u, v) K_{n-1}(x, y, u, v) d u d v
$$

we will have

$$
\left|S_{1}\right| \leq \iint_{G} h(u, v)\left|Q_{n, k}(u, v)\right|\left|K_{n-1}(x, y, u, v)\right| d u d v .
$$




\section{BRANISLAV FTOREK — PAVOL ORŠANSKÝ}

Now, we need to estimate the kernel $K_{n-1}(x, y, u, v)$. Using (4.2) and Cauchy inequality, we get

$$
\begin{aligned}
\left|K_{n-1}(x, y, u, v)\right| & \leq \sum_{m=0}^{n-1} \sum_{s=0}^{m}\left|F_{m, s}(x, y) F_{m, s}(u, v)\right| \\
& =\sum_{m=0}^{n-1} \sum_{s=0}^{m}\left|p_{m-s}(x) q_{s}(y) p_{m-s}(u) q_{s}(v)\right| \\
& \leq \sum_{i=0}^{n-1}\left|p_{i}(x) p_{i}(u)\right| \sum_{i=0}^{n-1}\left|q_{i}(y) q_{i}(v)\right| \\
& \leq\left(\sum_{i=0}^{n-1} p_{i}^{2}(x) \sum_{i=0}^{n-1} p_{i}^{2}(u) \sum_{i=0}^{n-1} q_{i}^{2}(y) \sum_{i=0}^{n-1} q_{i}^{2}(v)\right)^{\frac{1}{2}} .
\end{aligned}
$$

With regard to (4.4) and (4.5), we have

$$
\left|K_{n-1}(x, y, u, v)\right| \leq\left(K_{n-1}(x, x) K_{n-1}(u, u) K_{n-1}^{*}(y, y) K_{n-1}^{*}(v, v)\right)^{\frac{1}{2}} .
$$

From (4.12) and (4.13), the inequality

$$
\begin{aligned}
\left|S_{1}\right| \leq & \left(K_{n-1}(x, x) K_{n-1}^{*}(y, y)\right)^{\frac{1}{2}} \\
& \times \iint_{G} h(u, v)\left|Q_{n, k}(u, v)\right| K_{n-1}^{\frac{1}{2}}(u, u) K_{n-1}^{* \frac{1}{2}}(v, v) d u d v
\end{aligned}
$$

follows. To estimate this integral, we use (4.6) and we obtain

$$
\begin{aligned}
\left|S_{1}\right| \leq & \left(K_{n-1}(x, x) K_{n-1}^{*}(y, y)\right)^{\frac{1}{2}} \frac{1}{\sqrt{M}}\left(\iint_{G} h(u, v) \delta(u, v) Q_{n, k}^{2}(u, v) d u d v\right)^{\frac{1}{2}} \\
& \times\left(\iint_{G} h(u, v) K_{n-1}(u, u) K_{n-1}^{*}(v, v) d u d v\right)^{\frac{1}{2}} .
\end{aligned}
$$

Because the weight function $h(x, y)$ has separated variables (4.1), we replace the last integral with double integrals, and from orthonormal property of the systems $\left\{p_{n}(x)\right\},\left\{q_{n}(x)\right\}$, we have

$$
\begin{aligned}
& \iint_{G} h(u, v) K_{n-1}(u, u) K_{n-1}^{*}(v, v) d u d v \\
& =\int_{a}^{b} v_{1}(u) K_{n-1}(u, u) d u \int_{c}^{d} v_{2}(v) K_{n-1}^{*}(v, v) d v=n \cdot n=n^{2} .
\end{aligned}
$$


So,

$$
\left|S_{1}\right| \leq\left(K_{n-1}(x, x) K_{n-1}^{*}(y, y)\right)^{\frac{1}{2}} \frac{n}{\sqrt{M}} .
$$

Denoting the second integral in the expression (4.11) by $S_{2}$, we have

$$
S_{2}=\sum_{s=0}^{k} F_{n, s}(x, y) \iint_{G} h(u, v) Q_{n, k}(u, v) F_{n, s}(u, v) d u d v .
$$

Using condition (4.6), we obtain

$$
\left|S_{2}\right| \leq \frac{1}{\sqrt{M}} \sum_{s=0}^{k}\left|F_{n, s}(x, y)\right| \iint_{G} h(u, v) \sqrt{\delta(u, v)}\left|Q_{n, k}(u, v)\right|\left|F_{n, s}(u, v)\right| d u d v,
$$

and by Schwarz inequality,

$$
\begin{aligned}
\left|S_{2}\right| \leq & \frac{1}{\sqrt{M}} \sum_{s=0}^{k}\left|F_{n, s}(x, y)\right|\left(\iint_{G} h(u, v) \delta(u, v) Q_{n, k}^{2}(u, v) d u d v\right)^{\frac{1}{2}} \\
& \times\left(\iint_{G} h(u, v) F_{n, s}^{2}(u, v) d u d v\right)^{\frac{1}{2}} .
\end{aligned}
$$

From orthonormal properties of the systems $\left\{F_{n, m}(x, y)\right\}$ and $\left\{Q_{n, m}(x, y)\right\}$,

$$
\left|S_{2}\right| \leq \frac{1}{\sqrt{M}} \sum_{s=0}^{k}\left|F_{n, s}(x, y)\right|
$$

follows. From (4.11), we get

$$
\left|Q_{n, k}(x, y)\right| \leq\left|S_{1}\right|+\left|S_{2}\right|
$$

Finally, from (4.14), (4.15), we get the statement of the theorem.

\section{REFERENCES}

[1] DUNHAM, J.: Fourier Series and Orthogonal Polynomials. Dover Publications, Mineola, NY, 2004.

[2] DUNKL, CH. F.-XU, Y.: Orthogonal Polynomials of Several Variables, in: Encyclopedia Math. Appl., Vol. 81, Cambridge Univ. Press, Cambridge, 2001.

[3] JACKSON, D.: Fourier Series and Orthogonal Polynomials. Carus Math. Monogr., Vol. VI, Math. Assoc. America, Washington, DC, 1941.

[4] KOORNWINDER, T. H.: Orthogonal polynomials in two variables which are eigenfunctions of two algebraically independent partial differential operators I, II, Indag. Math. 36 (1974), 48-66. 


\section{BRANISLAV FTOREK — PAVOL ORŠANSKÝ}

[5] KOORNWINDER, T. H.: Orthogonal polynomials in two variables which are eigenfunctions of two algebraically independent partial differential operators III, IV, Indag. Math. 36 (1974), 357-381.

[6] KOROUS, J.: On the development of functions of one real variable in the certain series of orthogonal polynomials, Rozpravy II. třídy České akademie věd v Praze 40 (1938), 1-12. (In Czech)

[7] KRALL, H. L.-SHEFFER, I. M.: Orthogonal polynomials in two variables, Ann. Mat. Pura Appl. (4) 76 (1967), 325-376.

[8] MARČOKOVÁ, M.: Second order partial differential equations for some orthogonal polynomials in two variables, Stud. Univ. Žilina Math. Phys. Ser. 13 (2001), 127-132.

[9] MARČOKOVÁ, M.: Approximation of functions in two variables by Cesàro means of Fourier-Jacobi sums, in: Proc. of the Internat. Scientific Conf. of Mathematics, Vol. 1, (P. Marušiak et al., eds.), Žilina, Slovakia, 1998, EDIS, Žilina University Publisher, Žilina, 1999, pp. 161-165.

[10] MARČOKOVÁ, M.-GULDAN, V.: On one orthogonal transform applied on a system of orthogonal polynomials in two variables, J. Appl. Math.-Aplimat 2 (2009), pp. 239-245.

[11] SUETIN, P. K.: Orthogonal Polynomials in Two Variables, in: Anal. Methods Spec. Funct., Vol. 3, Gordon and Breach Sci. Publ., Amsterdam, 1999.

[12] SZABEOWSKI, P. J.: On affinity relating two positive measures and the connection coefficients between polynomials orthogonalized by these measures, Appl. Math. Comput. 219 (2013), 6768-6776.

[13] SZEGÖ, B.: Orthogonal Polynomials. Nauka, Moscow, 1962. (In Russian)

Received January 10, 2012

\author{
Branislav Ftorek \\ Pavol Oršanský \\ Department of Applied Mathematics \\ Faculty of Mechanical Engineering \\ University of Žilina \\ Univerzitná 8215/1 \\ SK-010-26 Žilina \\ SLOVAKIA
}

E-mail: branislav.ftorek@fstroj.uniza.sk pavol.orsansky@fstroj.uniza.sk 